Annika Niemann*, Bernhard Preim and Sylvia Saalfeld

\title{
Shrinking tube mesh: combined mesh generation and smoothing for pathologic vessels
}

https://doi.org/10.1515/cdbme-2020-0035

\begin{abstract}
We present a mesh generation algorithm which is able to produce smooth meshes from point clouds derived from histological slices. In this work, the shrinking tube mesh generation is used on histologic images depicting pathologic vessels. Our mesh generation is modeled after the behaviour of a shrinking tube. A start shape is fitted iteratively to the point cloud. The presented algorithm was successfully used to generate meshes of the inner and outer contour from vessels in histologic images. While histologic slices have a high in-plane resolution, the large slice distance and deformations during tissue deformations are challenging for 3D model generation.
\end{abstract}

Keywords: histology; intracranial aneurysm; mesh generation.

\section{Introduction}

3D models from medical images are commonly used to support diagnosis and treatment decisions. Using 2D histologic images we want to generate a 3D model for visualisation and simulation. Several model-based algorithms for mesh generation from medical images exist [2-4]. Frangi et al. [3] developed an algorithm using a deformable model to derive vessel models from 3D magnetic resonance angiograms. Similar, Yim et al. [4] used a tubular deformable model to reconstruct vessels. While providing good results, these algorithms are restricted to the specific use case (imaging modality and organ) they were designed for.

Due to several reasons, mesh generation is especially challenging when working with histologic images. The images are very large (approximately $11,000 \times 8,000$ pixel)

*Corresponding author: Annika Niemann, Faculty of Computer Science, Otto-von-Guericke University Magdeburg, Magdeburg, Germany, E-mail: annika.niemann@ovgu.de Bernhard Preim, Faculty of Computer Science, Otto-von-Guericke University Magdeburg, Magdeburg, Germany

Sylvia Saalfeld, Faculty of Computer Science \& Research Campus STIMULATE, Otto-von-Guericke University Magdeburg, Magdeburg, Germany and several artefacts can influence the tissue shape (folding, dissecting tissue, deformations during tissue collections). The first problem is addressed by working with the points of the outer contour of the tissue instead of the images. By registering the images and extracting the contour points, a point cloud is derived. The point cloud has a very anisotropic distribution (close points in $x-y$-direction, large gaps along the $z$-axis) and due to the artefacts it does not correctly represent the outer aneurysm border. Here, a process to generate smooth meshes from noisy pathologic vessel point clouds is described. In contrast to healthy vessels the pathologic vessels used here can only be roughly approximated by a cylinder and do not allow for more detailed assumptions to guide the model generation.

Salman et al. [5] proposed a mesh generation from point clouds, which includes a feature selection step before the mesh generation. The approach preserves sharp edges well and is therefore suitable for objects with sharp edges like buildings or technological components. Often the meshes derived from point clouds require a post processing step to smooth the meshes [6]. Some algorithms require extensive preprocessing, for example the calculation of point normals [7]. Although this preprocessing is justified, the normals are error-prone in case of incorrect segmentations. Mostly, a point cloud is available consisting of points which are nearly evenly spaced over the object, for example from a 3D scan of an object. In the images the inner and outer contour is segmented. To get a 3D point cloud of these contour points, a $z$-coordinate based on the slide number and distance between slides is added. Due to the high resolution of histolgic images, the reduction to contours and point clouds allows for a more efficient processing. Figure 2 shows an exemplary case of our data, a point cloud of segmented histologic slices. Hence, the equal distribution criterion is not fulfilled yielding a problematic result of the Poisson surface reconstruction [8]. The ball pivoting algorithm [9] only produces a few faces along the points of one slice and cannot handle the large distance between points from different slices.

We use the knowledge of the elongated structure of vessels to generate the 3D model. While being robust against the challenges of histologic images (large slicedistance, artefacts distorting the border) our algorithm does not use image specific information and therefore can 
be applied to different histologic stainings or other image modalities. The presented algorithm models the behaviour of a shrinking tube., as illustrated in Figure 1A shrinking tube is plastic tube which can be used to isolate wires and is tightly fitted to them by applying heat.

\section{Shrinking tube mesh generation}

We consider three different possibilities for the start shape: a straight cylinder $\left(C_{\mathrm{S}}\right)$, the convex hull of the point cloud (CH) or a cylinder based on the maximum diameter of each slice $\left(C_{\max }\right)$. For $C_{\max }$, the maximum diameter of each slice is determined. A cubic smoothing spline is fitted through these values yielding a function $f(x)$. Next, $f(x)$ is rotated around the $x$-axis to get a cylinder. This can be varied by smoothing $f(x)$ which will result in a smoother cylinder. The choice of the optimal start shape depends on the point cloud and expected shape of the structure. $C_{\mathrm{S}}$ results in a smooth mesh and is able to avoid the generation of pseudostenosis due to poor image quality or segmentation. It is only suitable for elongated structures which can be approximated with a cylinder, like a vessel. $C_{\max }$ better preserves the underlying structure and is better suited when variation is expected, like plaque-ridden vessel walls. $\mathrm{CH}$ is suitable for a large range of point clouds and is not limited to cylinder-like structures. In contrast to the cylinders, the $\mathrm{CH}$ is at higher risk to contain sharp edges.

The mesh is then fitted to the point cloud. This step has three parameters. In analogy to a shrinking tube (Figure 1), the first two parameters are the number of time steps (iterations) and the temperature (speed). The third parameter is the influence area factor. This factor describes how many vertices a point of the point cloud influences. In each iteration, the points of the mesh are moved closer to the point cloud. How far the mesh points are moved in direction of the point cloud depends the temperature and the distance of the point to the mesh. The factor for the displacement based on the distance is calculated using a quadratic function. Small distances have only a small impact, as they are likely noise and very large distance have a small impact on the mesh as they are expected to be artefacts of the segmentation. For each mesh point, the

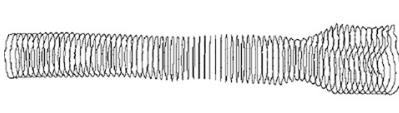

(a)

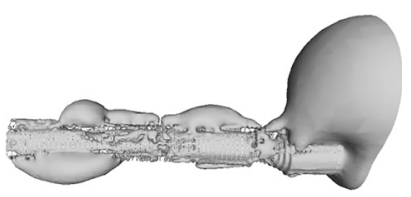

(b)

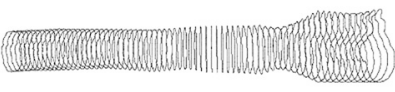

(c)

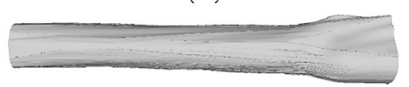

(d)

Figure 2: (a) Point cloud from vessel contours. (b) The result of MeshLabs screened poisson surface reconstruction algorithm. (c) The result of MeshLabs ball pivoting algorithm, triangles are only generated between points derived from the same slide; both algorithms have major problems with the uneven distribution of the points. (d) The mesh generated by our shrinking tube mesh algorithm [9].

closest point of the point cloud is determined and the mesh point is moved in direction of this point. Then the neighbours of the mesh point are moved in the direction of the new position of the mesh point. This is iterative repeated for the neighbours of the neighbours, depending on the distance from the point cloud and the influence area factor. The start shape and the number of vertices in the start shape have a large influence on the resulting mesh. The results of different start shapes are shown in Figure 4. Using $\mathrm{CH}$ the fitting progress is faster. After sufficient time steps, $C_{\mathrm{S}}$ and $C_{\max }$ will converge to similar results.

\section{Experiments}

Next, the influence of the parameters are analysed. While allowing for adaption to different problems and point clouds, the algorithm has a large number of parameters and optimising these can be time consuming. Here we show the results of different parameters for one point cloud. For each vertex of the mesh the distance to the closest point of the point cloud is calculated. The sum of these distances estimates the distance between mesh and point cloud. The mesh should be close to the point cloud, therefore the added up distance should be low. As some noise in the point cloud is expected, the distance should be non-zero as the mesh should be smooth. Additionally, the

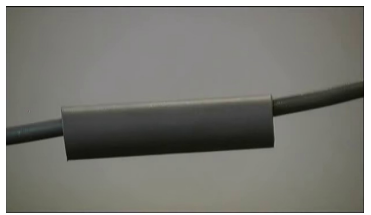

(a)

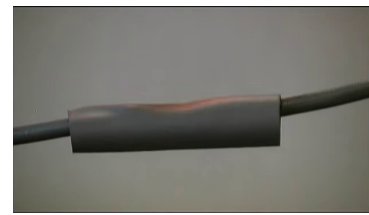

(b)

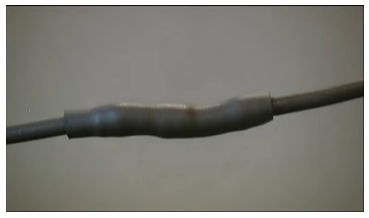

(c)
Figure 1: (a) Shrinking tube, (b) during heat, (c) fitted shrinking tube [1] 
calculation time is measured. Varying only the temperature (between 0 and 1), the difference between the points of the mesh and the nearest points of the point cloud decreases as shown in Figure 5. A larger influence area leads to a smoother, closer to the points fitted mesh. The time needed for the shrinking tube mesh generation increases with the influence area factor as shown in Figure 5 (Experiments were run on a computer with an Intel i7 processor with 16 GB RAM). While the time linearly increases with the number of time steps, the mesh does not improve linearly (Figures 3 and 5). As a result, the optimal values for the parameters depend on the point cloud shape, target structure and the number of vertices of the start shape. Therefore, no general recommendation for parameter settings can be given.

The movement of a vertex depends on the temperature parameter and the distance from the vertex to the point cloud. The connection between distance to the point cloud and movement of the vertex is described by the moving factor function. As mentioned above, a small distance (likely noise) or a very large distance (likely artefact) should lead to small vertex movements.

\section{Discussion}

The selection of the start shape has a large influence on the produced mesh. With a higher temperature or higher number of time steps the mesh is closer fitted to the point cloud. The optimal fit depends on the individual application and quality of the point cloud. The moving factor function indirectly describes the expected noise in the point cloud and should be chosen appropriately.

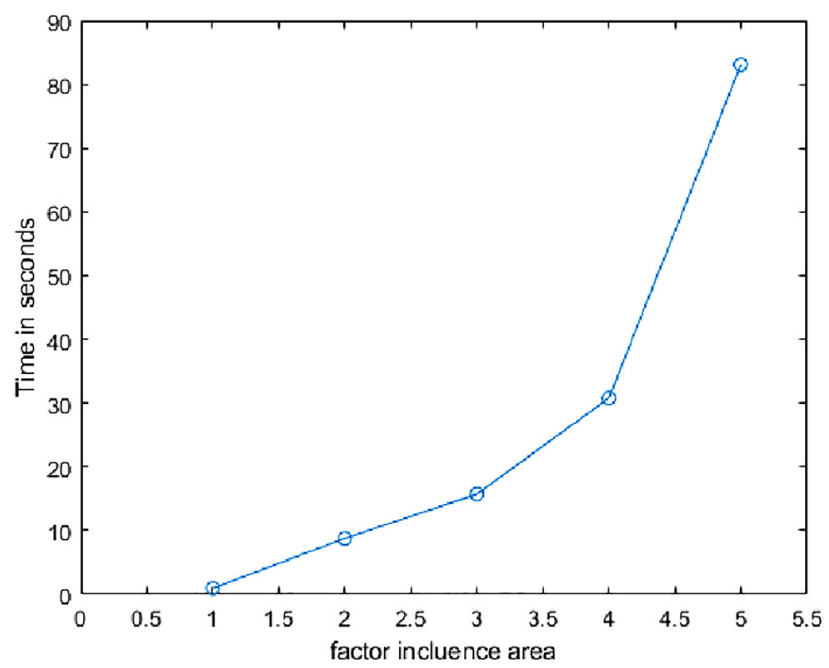

Figure 3: Increasing of calculation time with increasing influence area.

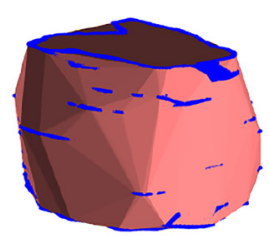

(a)

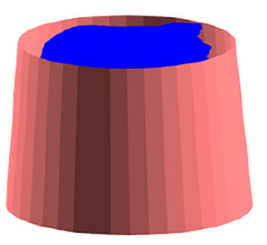

(c)

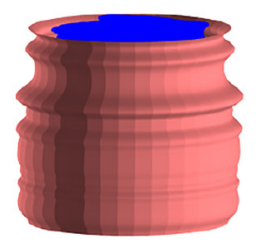

(e)

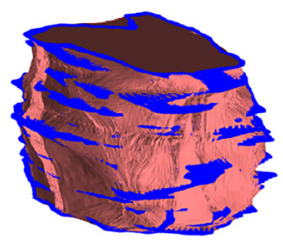

(b)

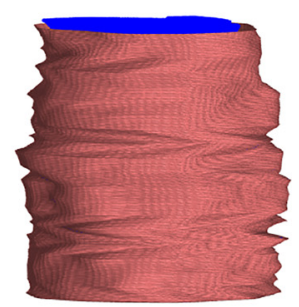

(d)

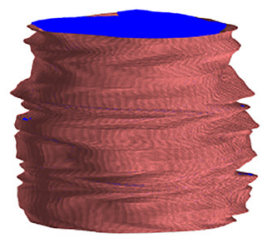

(f)
Figure 4: Start shapes and behaviour: (a) Start shape: $\mathrm{CH}$, (b) corresponding result, (c) Start shape: $C_{\mathrm{S}}$, (d) corresponding result, (e) Start shape: $C_{\max }$, (f) corresponding result.

The shrinking tube mesh generation produces smooth meshes without additional postprocessing. It can be applied to noisy segmentations. Compared to image stacks, point clouds from contours requiere less memory. The algorithm is robust against uneven distribution of points. There is a large number of factors which influence the behaviour and outcome of the algorithm. With enough time steps or a high value for the temperature parameter the resulting mesh will be tightly fitted to the point cloud. As it was developed for medical applications, where sharp edges rarely occur, it might not preserve these. Future work could include an interactive mesh improvement. The algorithm does make some assumptions about the relevance of the points. The influence of the points is based on their distance to the mesh. Finally, we were able to successfully generate meshes from the inner and outer contour of our histologic data yielding inner and outer vessel walls with the presented approach. 


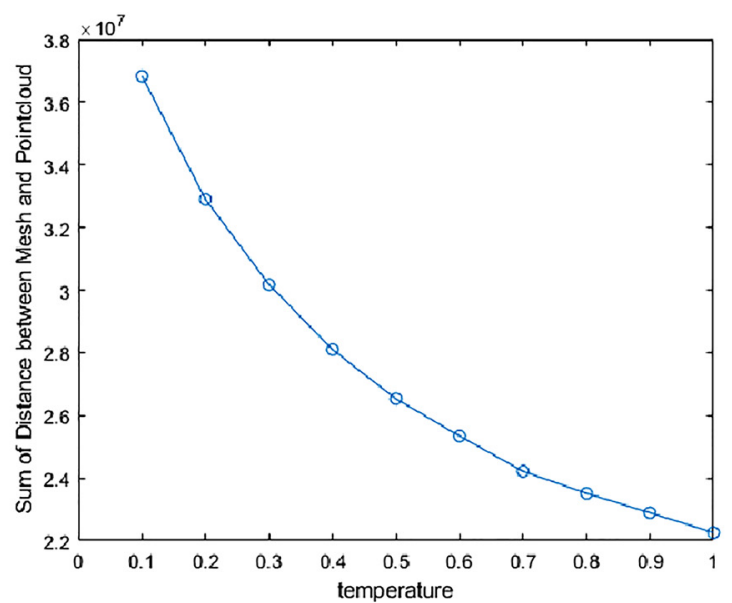

(a)

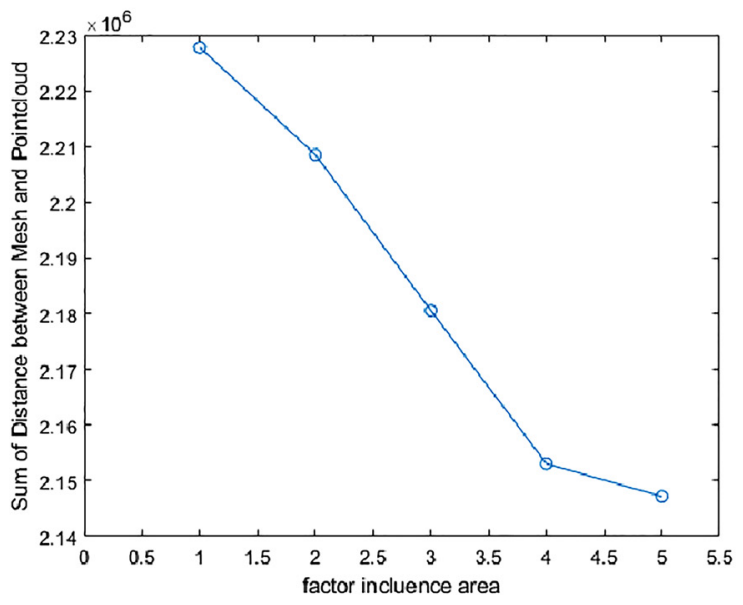

(b)

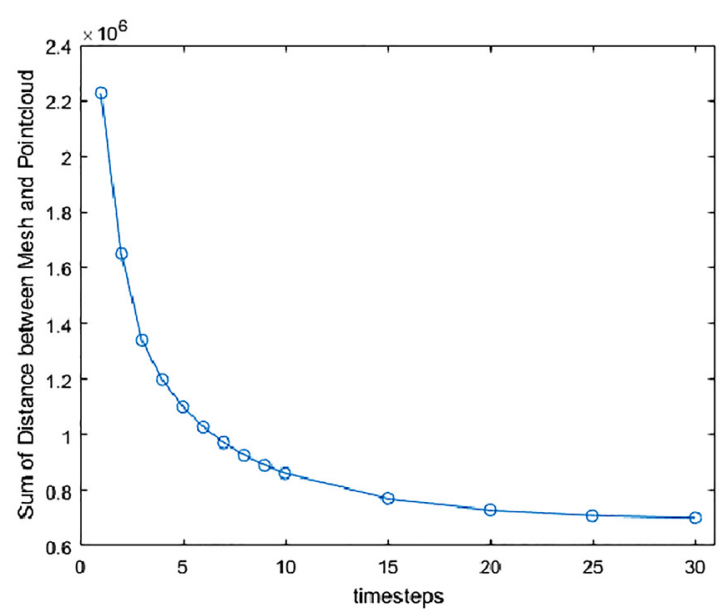

(c)

Figure 5: Influence of parameter on the fitting of the mesh to the point cloud: (a) temperature, (b) factor influence area, (c) timesteps.

\section{Conclusion}

The presented approach is suitable to generate smooth meshes from noisy point clouds derived from histologic images. It is developed for vessels and could be transferred to different image modalities. Several parameters influence the result and can lead to a smoother mesh or a mesh closely fitted to the point cloud.

Research funding: This work is partly funded by the German Research Foundation (SA 3461/2-1) and the Federal Ministry of Education and Research within the Forschungscampus STIMULATE (13GW0095A).

Informed consent: Informed consent has been obtained from all individuals included in this study.

Ethical approval: The research related to human use complies with all the relevant national regulations, institutional policies and was performed in accordance with the tenets of the Helsinki Declaration, and has been approved by the authors' institutional review board or equivalent committee.

Conflict of interest: Authors state no conflict of interest.

\section{References}

1. Video of heat shrink tube before and after https://commons. wikimedia.org/w/index.php?title=File\%3AVideo_of_Heat_ shrink_tube_before_and_after.ogv [accessed 30 Apr 2020].

2. Cebral JR, Löhner R. From medical images to anatomically accurate finite element grids. Int J Numer Methods Eng 2001;51: 985-1008.

3. Frangi AF, Niessen WJ, Hoogeveen RM, van Walsum T, Viergever MA. Model-based quantitation of 3-D magnetic resonance angiographic images. IEEE Trans Med Imaging 1999;18:946-56.

4. Yim PJ, Cebral JJ, Mullick R, Marcos HB, Choyke PL. Vessel surface reconstruction with a tubular deformable model. IEEE Trans Med Imaging 2001;20:1411-21.

5. Salman N, Yvinec M, Merigot Q. Feature preserving mesh generation from 3D point clouds. Comput Graph Forum 2010;29: 1623-32.

6. Lin H - W, Tai G - J, Wang C - L. A mesh reconstruction algorithm driven by an intrinsic property of a point cloud. Comput Aided Des 2004;36:1-9.

7. Woo H, Kang E, Wang S, Lee KH. A new segmentation method for point cloud data. Int J Mach Tools Manuf 2002; 42:167-78.

8. Kazhdan M, Hoppe H. Screened Poisson surface reconstruction. ACM Trans Graph 2013;32:1-13.

9. Cignoni P, Callieri M, Corsini M, Dellepiane M, Ganovelli F, Guido R. Meshlab: an open-source mesh processing tool. In: Eurographics Italian chapter conference; 2008, vol 1, pp. 129-36. 01. 